\title{
A Value-Stream Mapping Success Story: MBA Recruiting Process Improvements
}

\author{
Dr. Paulette S. Alexander \\ Email: psalexander@una.edu \\ Janyce Brennan Fadden \\ Email: jfadden@una.edu
}

University of North Alabama

\begin{abstract}
Purpose: The benefits of Value Stream Mapping (VSM) in higher education are highlighted through this case study which demonstrates that by using the practices of VSM, the graduate recruiting process can be successfully improved by engaging faculty and staff in change events and with rigorous follow up.
\end{abstract}

Design/Methodology/Approach: A University of North Alabama (UNA) cross-functional team built a sustainable MBA recruiting process, which resulted in improved enrollment metrics such as an average $70 \%$ enrollment increase. This case study details the MBA enrollment before and after the cross-functional team implemented VSM tools focused on improving prospective student engagement. UNA engaged in a multi-day planning and subsequent implementation process that included regular meetings to assess results.

Findings: VSM led to a student-centered process that meets adult learner online student needs within a traditional on-campus system. The team learned that consistent emphasis on the "future state" is more motivational to them than placing blame for the "current state." In addition, the team learned that they could not predict what they will find in the current process, as it had never been managed as one system. The analysis shows the changes over time in prospect to enrolled student yield rates for all stages, actions taken that improved results and the incorporation of continuous improvement thinking. This study demonstrates that universities can implement changes that positively affect student recruitment satisfaction and enrollment results.

Practical Implications: Recruiting graduate students in an era of declining demographics and transformational learning processes is putting many universities' MBA enrollment in jeopardy.

Originality/Value: This case study demonstrates the challenges universities face in changing old silo processes into fully interactive processes targeted to student-centered results using collaborative techniques. It also demonstrates and reinforces the idea that VSM is extremely applicable to and useful for institutions of higher learning.

Keywords: Customer Relationship Management (CRM), MBA recruitment, Value Stream Mapping, Yield rate, Waste

Paper type: Research 


\section{Background}

In July 2013, the UNA College of Business (COB) named a new dean who set a goal to increase the MBA enrollment. Nationally for several years, MBA enrollments were flat or in decline. UNA had also experienced a decline in enrollment. This trend has serious implications for universities, as MBA programs are known to be a key source of a COB's revenue.

One of the first steps taken by the Dean was to replace the external marketing partner, with a new partner who had strong experience in programs for adult learners and deep experience in digital pay-per-click (PPC) marketing. In addition, the recruiting function was brought inhouse and a new team was established. The new partner immediately asked for a process map. The response was that none existed.

Often in a university setting, the culture is about efficiency by reducing operational waste. True transformation occurs when the Value Stream is viewed in a broader way as a methodology to transform the thinking and culture to one focused on customer value. (Martin, 2014).

After exploring options, the Dean engaged the local Manufacturing Extension Partner (MEP) to lead a cross-functional team through the first 3-day VSM event in March 2014. Not only was this the first such event ever held on campus, but most of the team had never heard of lean management so the journey was new, exciting and challenging.

The team finished the event with a rudimentary VSM map (Chart 1) and a long list of action items.

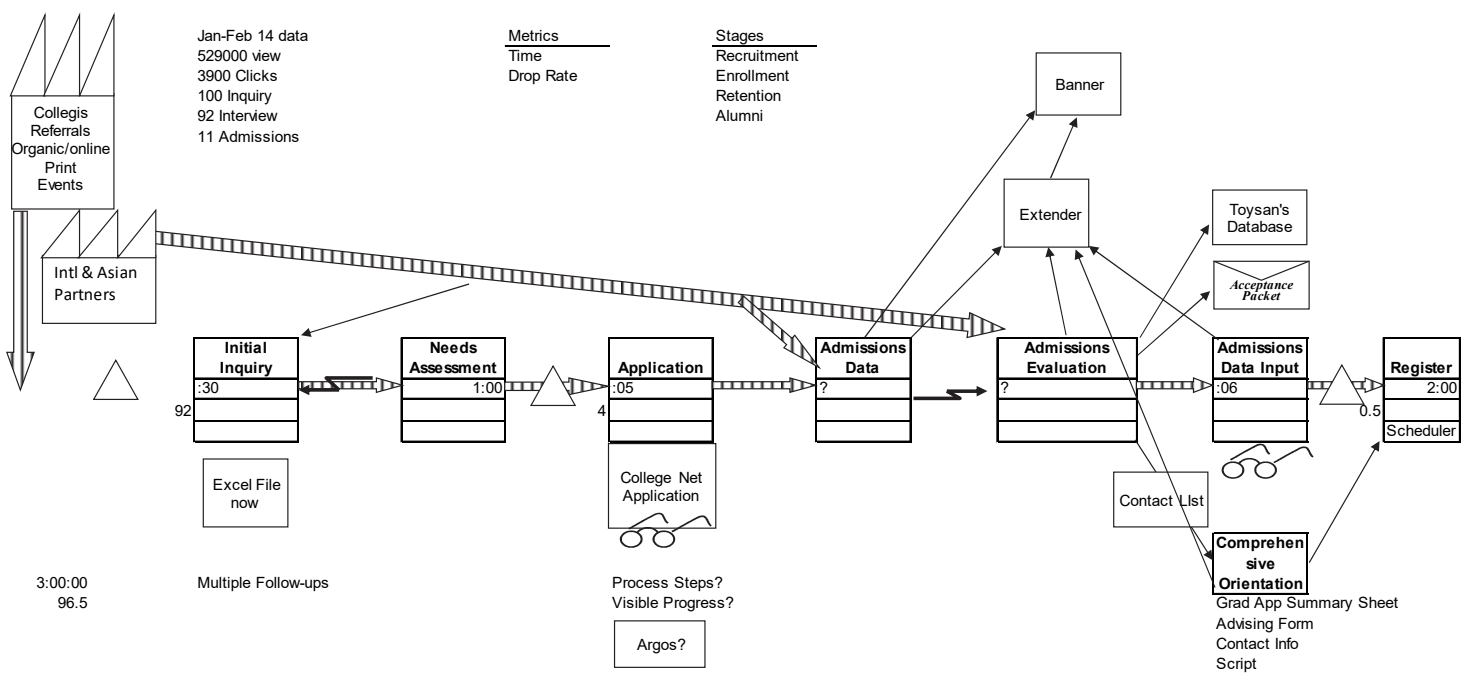

Chart 1. First VSM Map

For the next 3 years, regular follow-up meetings ensued where the team evaluated data and implemented actions to reduce waste. The Current State Process Map (Chart 2) now identifies five stages to enrollment including Prospecting, Nurturing, Application, Admission, and Registration. 


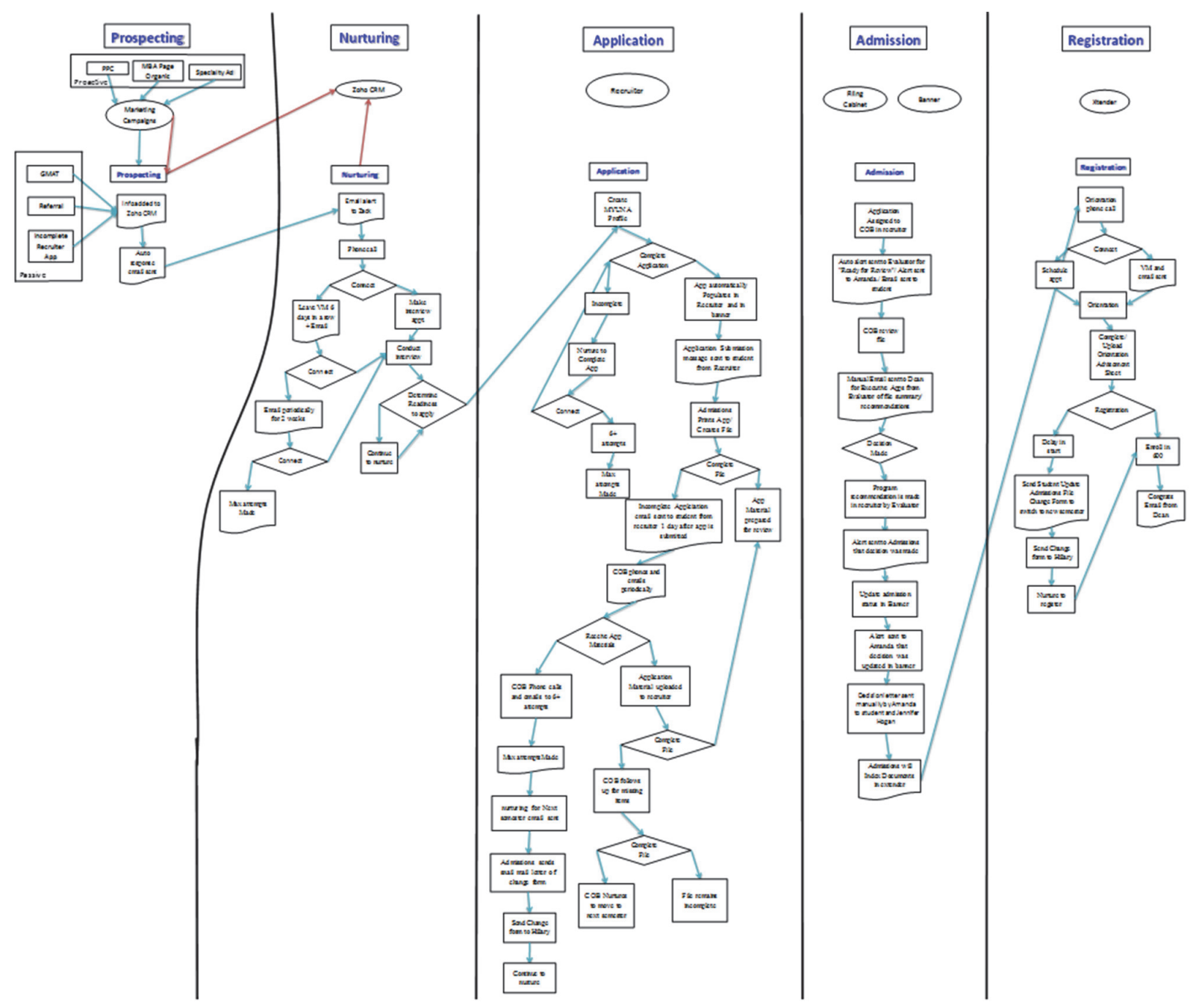

Chart 2. Current State Process Map

Until the team saw this process on one map, they had accepted the status quo of hockey-stick style enrollment patterns related to the university's three semesters where the enrollment pattern followed the shape of a hockey stick with almost all registrations happening in the last week of enrollment. This pattern caused much chaos at the start of the semester as students tried to enroll before the class cutoff date. Understanding that a pull system could boost productivity, minimize mistakes and increase enrollments, the team implemented changes to engage the prospective student to enroll sooner. Through leveling enrollments, the goals are met in a more consistent manner and the team is able to give each prospect adequate customer service time. Stopping any batching and understanding how any flow is hindered led to several actions. Without flow, the system is full of waste and potential errors. (Womack \& Jones, 1996).

After attending a training session (Picture 1) for Lean Management for Higher Education in December 2016, staff members initiated a Rapid Improvement Event (RIE) that allowed the cross functional team to put "fresh eyes" on the overall process. This resulted in the identification of 25 improvement action items. 


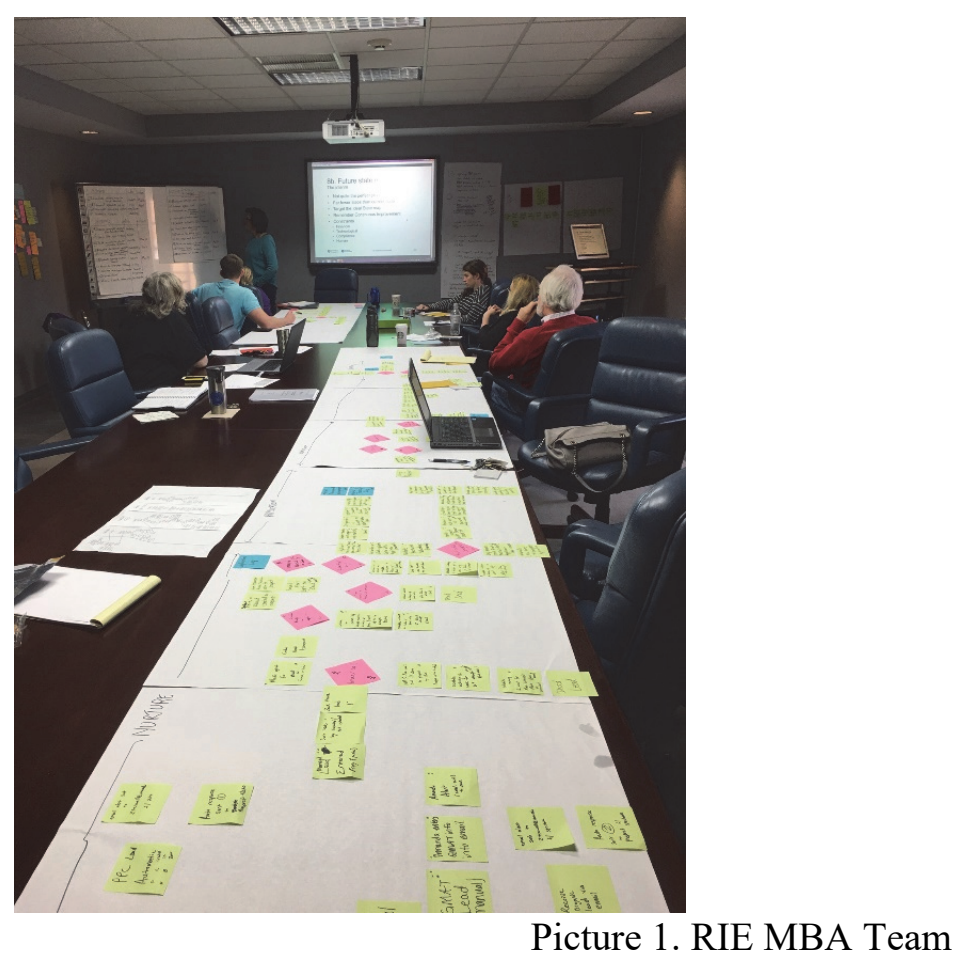

The RIE event created Interim and Ideal state maps that now drive improvements (Chart 3 and 4). Currently, changes to achieve the interim state map are underway as the team aims for perfection to the ideal state map.
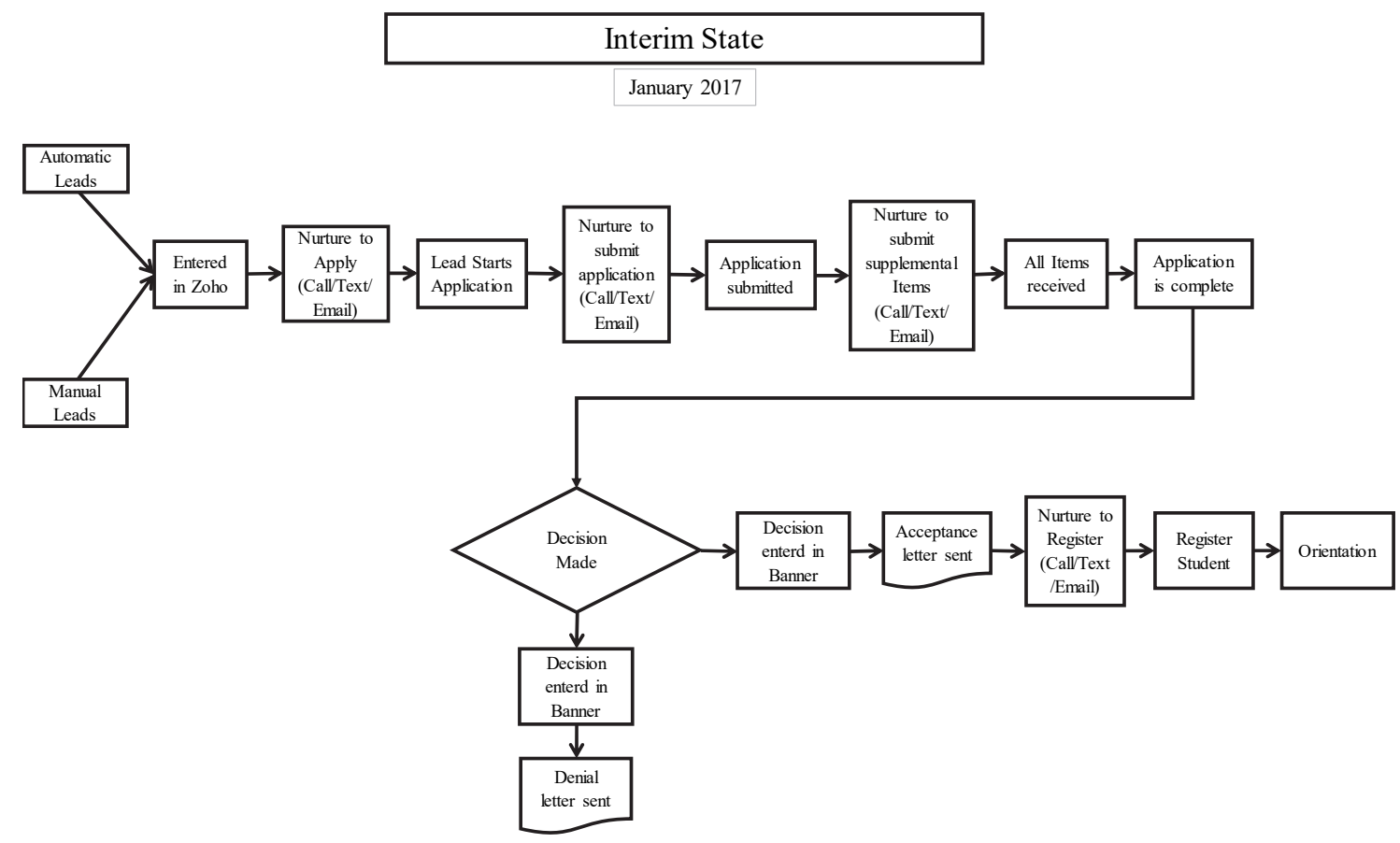

Chart 3. RIE Interim State Map 


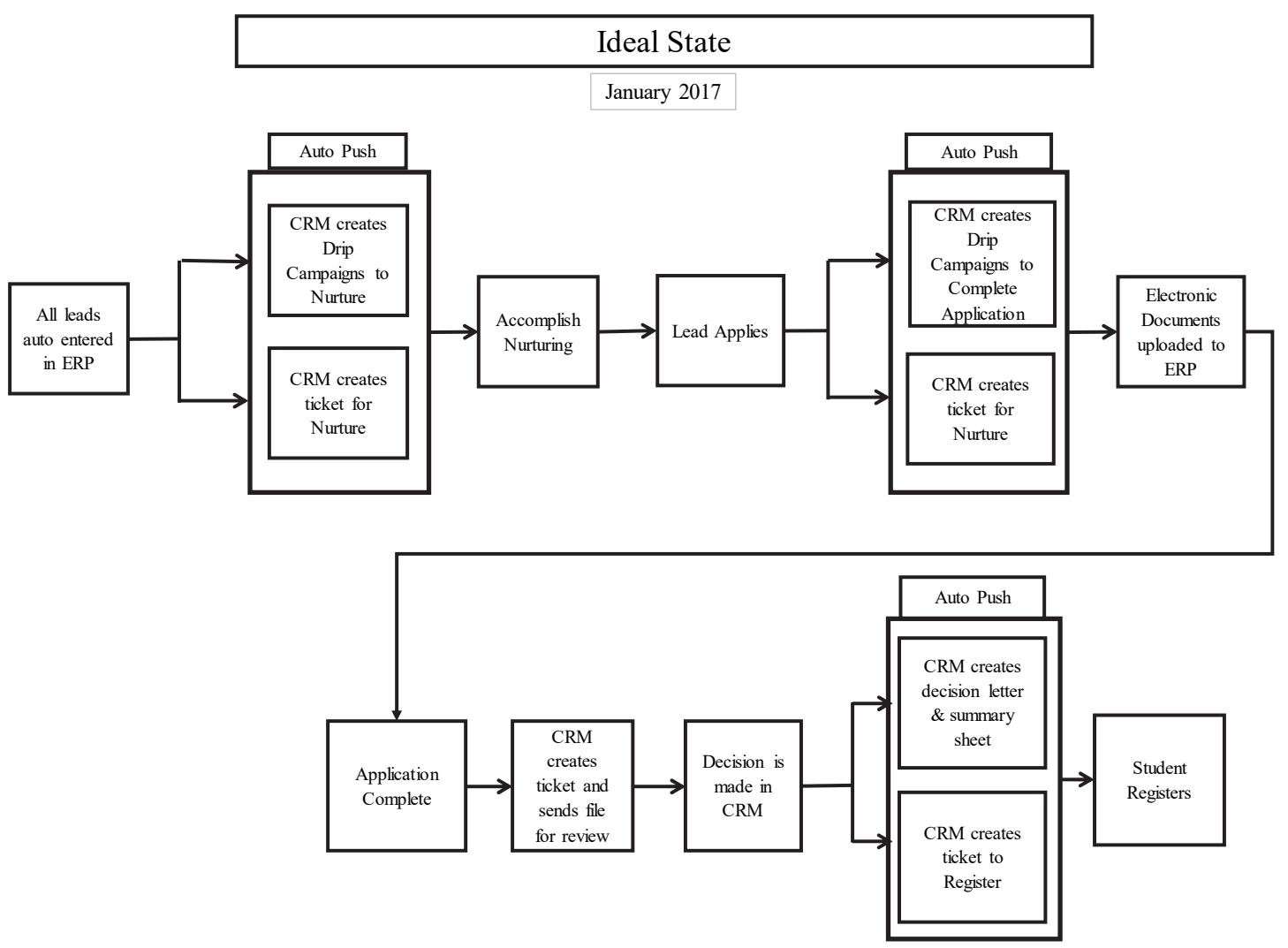

\section{Hypothesis}

Chart 4. RIE Ideal State Map

Our hypothesis is that by improving the overall MBA recruiting process through a collaborative systematic review instead of an individual silo review, the program would gain a significant increase in student enrollment and satisfaction.

1. Asking ourselves- what would it look like if every one of our actions added value to the student? What could we do? Do we have repetitive steps? Do we have unseen barriers? Do we have smooth, seamless transitions?

2. Could we organize around a process instead of silos to achieve higher student satisfaction and enrollment?

3. Can we increase MBA enrollments through using the VSM lean methodology?

4. Would the dean, staff, faculty, and university administration support the work of using VSM and the process changes that could result from the analysis?

\section{Methodology}

The team began in March 2014 with VSM training by the MEP, whose curriculum is manufacturing focused. This made the mapping more difficult as the tools had a decided slant to "widget production" instead of "student recruitment". The training definitely introduced new thinking and language such as cross training, pull vs push, only one way, 8 forms of waste, 5 whys, and current vs future state.

The multi-day event was the beginning of the transformation. The team established regular meetings including: 
1. Weekly MBA enrollment funnel meetings

2. Monthly digital marketing review meetings

3. Monthly VSM update and action planning

4. Semester review meeting with in-depth analysis

A key asset implemented was the use of Customer Relationship Management (CRM) software that offered great flexibility. The CRM provides visibility on the current state of each prospect as well as the next steps to pull the prospective student through the stages to registration.

In January 2017, the team conducted a 3-day RIE, the results of which now drive their future actions. One identified area of improvement was to connect wireless headsets, mobile phones and email to the CRM, which builds team capability to know all communications and assists pulling the prospective student towards registration.

\section{Results and Analysis}

The goal of the VSM project is to increase enrollment and student recruiting satisfaction. Enrollment grew over 70\% from the 2012/13 year to 2015/16 with an estimated growth of $54 \%$ from $2015 / 16$ to $2016 / 17$. Student MBA recruitment satisfaction is at $97 \%$ for agreeing or highly agreeing.

GMAC (Graduate Management Admission Council, 2015) reported a 2 year average for prospect to student enrollment. Since the pipeline was not tracked previously, our results began to appear at the two-year mark in 2015/16 (Chart 5). Enrollment definition is the first time the student registers in the MBA program.

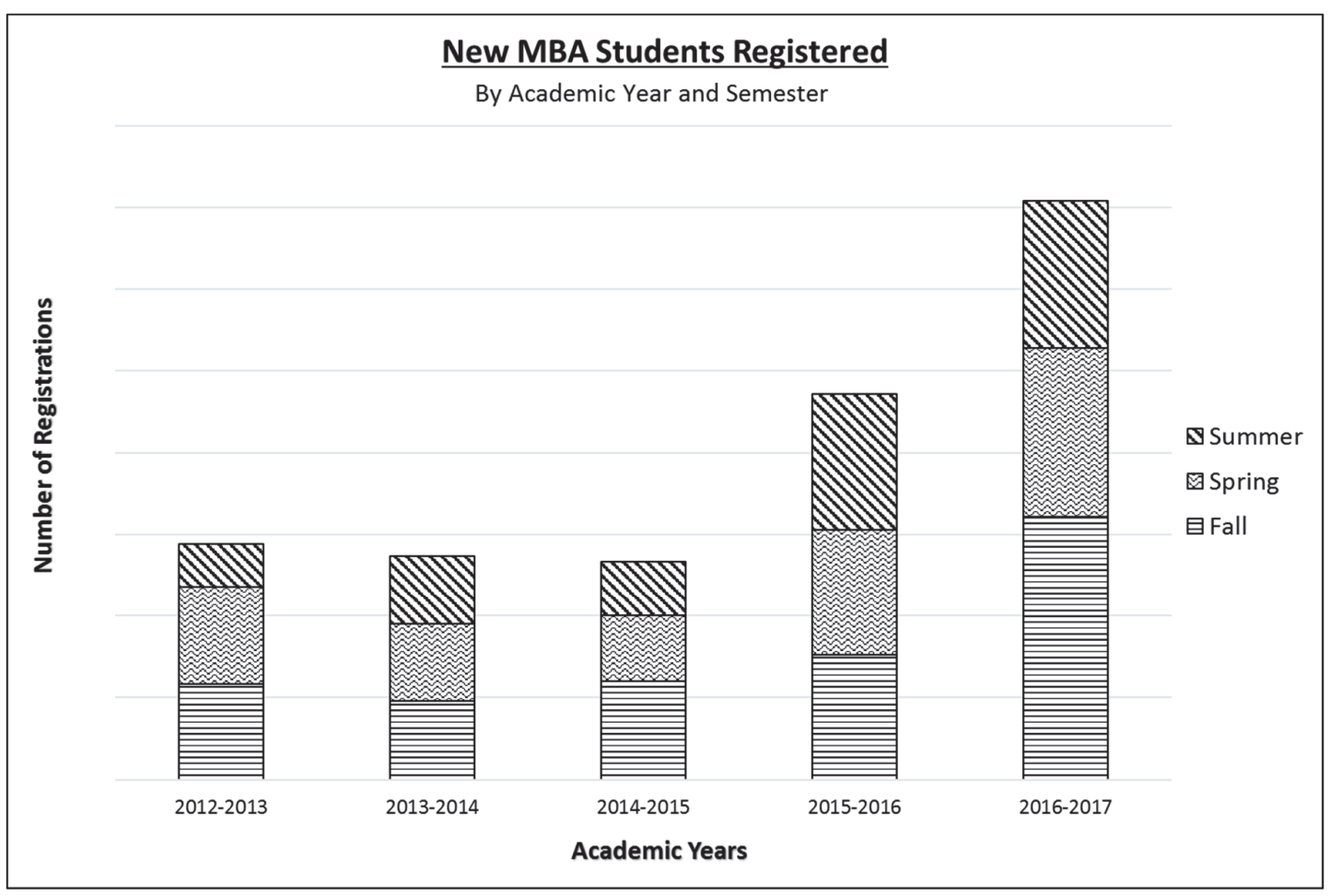

Chart 5. New Student Starts 
A great indicator of successful growth is the yield rate by process stage. Each semester the yield rate is carefully tracked and observed to assure goals are met or exceeded, to explain any anomalies, and to look for areas to impact performance. The yield rates have consistently improved converting more leads to enrollment, which greatly improved our ability to estimate the next semester's potential registrations (Table1).

\begin{tabular}{|l|r|r|r|}
\hline Semester & Leads to Apps & Apps to Admits & Admits to Registered \\
\hline Fall 2014 & & $55 \%$ & $72 \%$ \\
\hline Spring 2015 & $51 \%$ & $51 \%$ & $48 \%$ \\
\hline Summer 2015 & $41 \%$ & $75 \%$ & $53 \%$ \\
\hline Fall 2015 & $52 \%$ & $55 \%$ & $69 \%$ \\
\hline Spring 2016 & $47 \%$ & $53 \%$ & $81 \%$ \\
\hline Summer 2016 & $55 \%$ & $53 \%$ & $92 \%$ \\
\hline Fall 2016 & $58 \%$ & $70 \%$ & $95 \%$ \\
\hline Spring 2017 & $59 \%$ & $67 \%$ & $71 \%$ \\
\hline
\end{tabular}

Table 1. Yield Rates

Tracking the total time to complete the MBA recruiting process versus the actual value added time has proven to be a very difficult measurement to reduce because many variables are outside the direct control of the recruiting team for example, completing the application or taking the entry exam (Chart 6.). The team identifies variables that can be managed to assure increased control and quality. Then data is analyzed to determine actions that could eliminate time between and within stages.

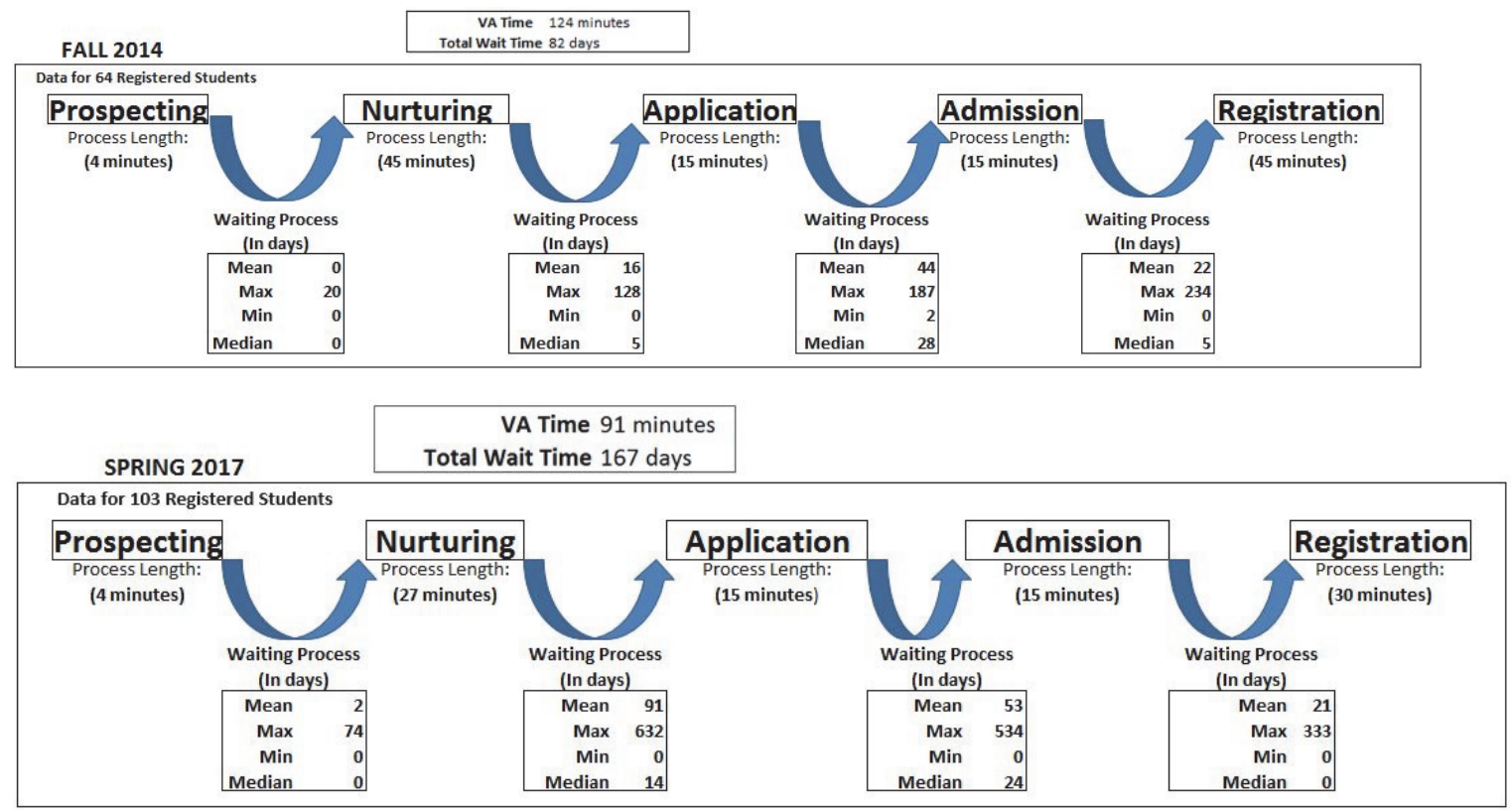

Chart 6. Value Added versus Non-value Added Time

One example of how the team discovered and used data is looking at BBA vs non-BBAdegreed students including the number in each stage (Chart 7). The team noted that a higher percentage of registered students have a BBA than a non-BBA degree. In evaluating the cause using "5 Whys" discovery concluded that non-BBA students were accepted with the condition that they pass an average of 6 prerequisite courses offered by our Office of 
Continuing Education. However, there was no follow-up to ascertain their progress through the prerequisites. That led to a data review on the students who successfully completed the prerequisites where we found that only 1 in 4 would eventually enroll in the MBA program. Several short-term actions to improve engagement with these accepted students were enacted and a small rise in students completing the courses and enrolling in the MBA program was observed. Longer term a newly formed faculty committee built a new course, MG600 Foundations of Business, which eliminates the need for prerequisites and begins all students in the same course. This key change introduced in summer 2016 has positively influenced registrations. In addition, the course is now for credit so it qualifies for employer reimbursement and loans if applicable.

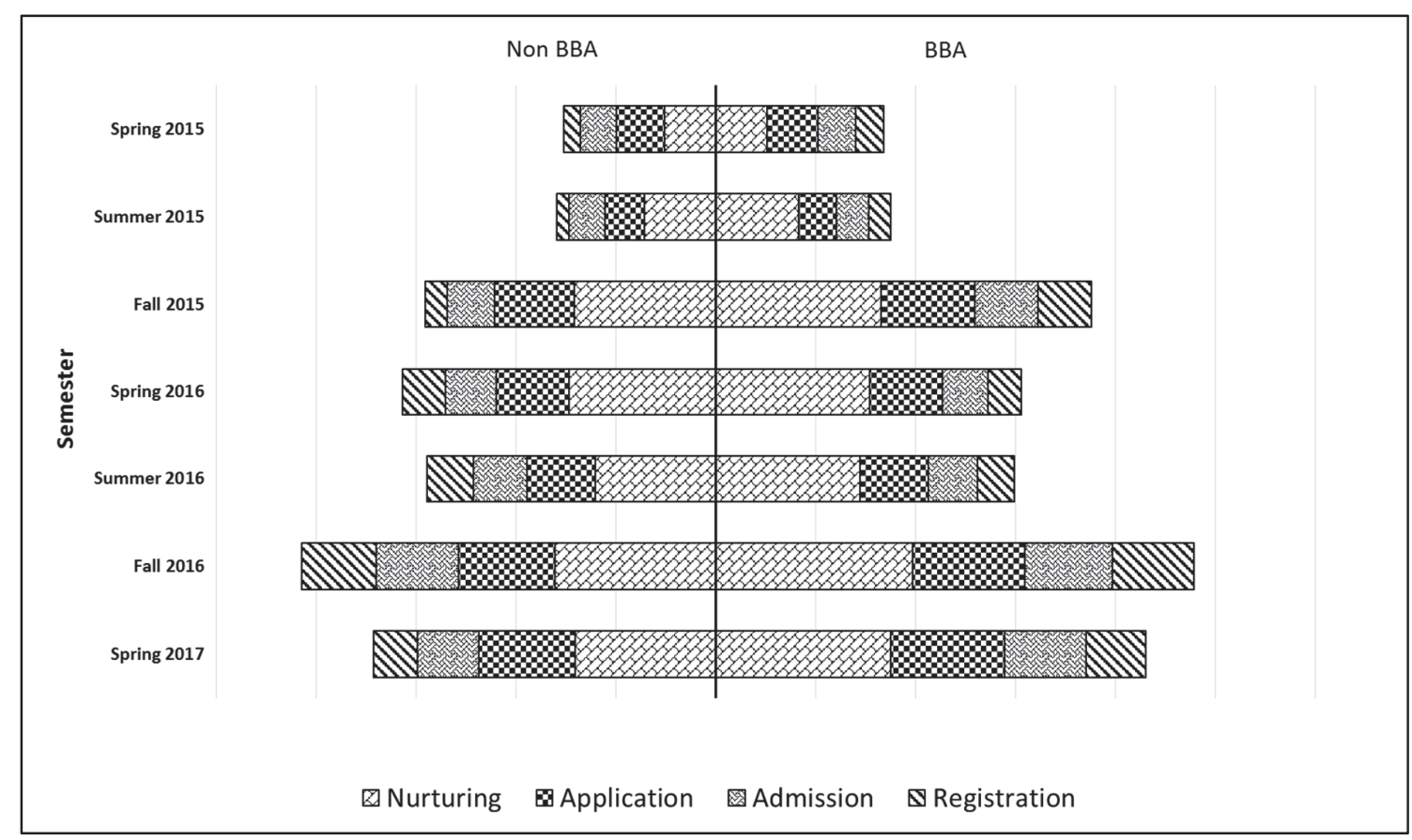

Chart 7. BBA versus Non-BBA Pipeline

Student satisfaction is assessed each semester in the first class, MG600 Foundations of Business and a recent example (Chart 8) shows a 97\% satisfaction level in the recruiting process. Evaluated each semester as student needs are always increasing the team applies VSM processes to determine improvements. The aim is perfection though endless steps which is a fundamental principle of lean thinking. (Womack \& Jones, 1996) 


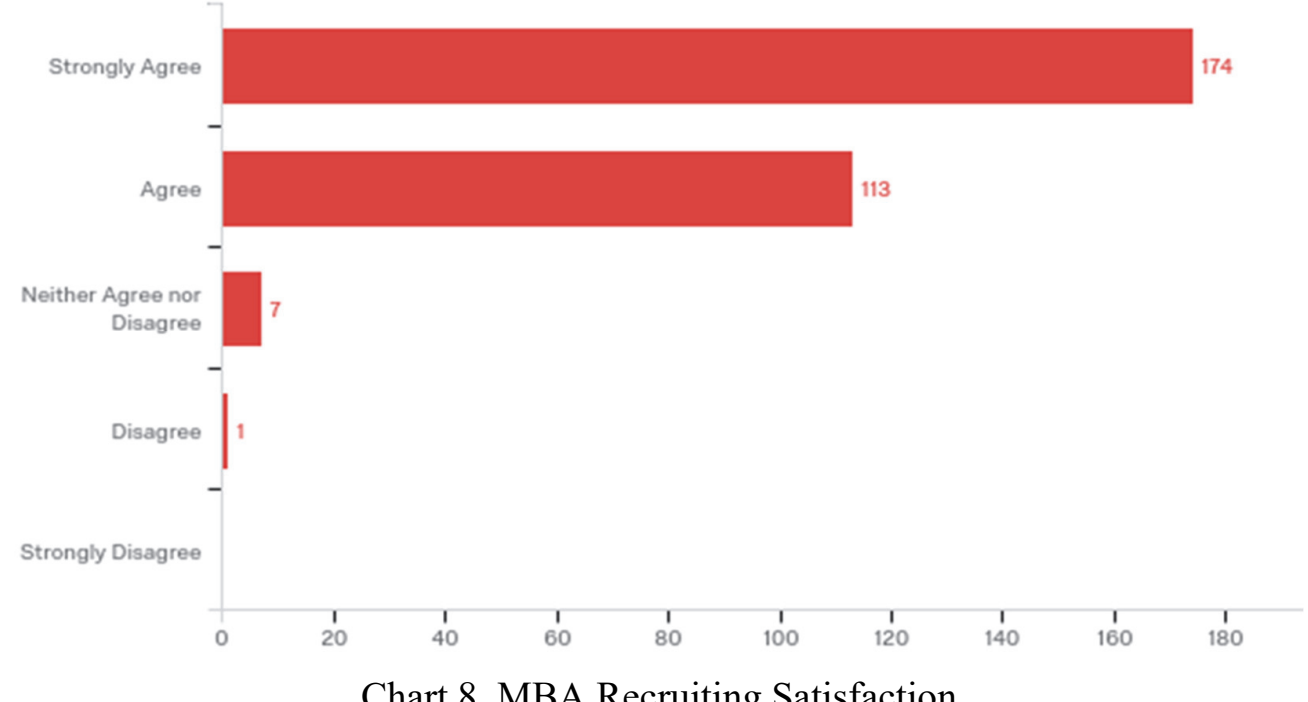

The MBA leads are primarily created through a successful pay-per-click program. The team reviews the spending on ads monthly, the click through rate, and cost per student. The trends are very positive for increasing conversion and lowering recruiting cost per enrolled student (Chart 9 calculated without showing actuals). By closely monitoring data, steps that would positively impact the enrollment are determined, prioritized and implemented. A cloud-based shared software stores the VSM action items so team members can see the status and record their progress.

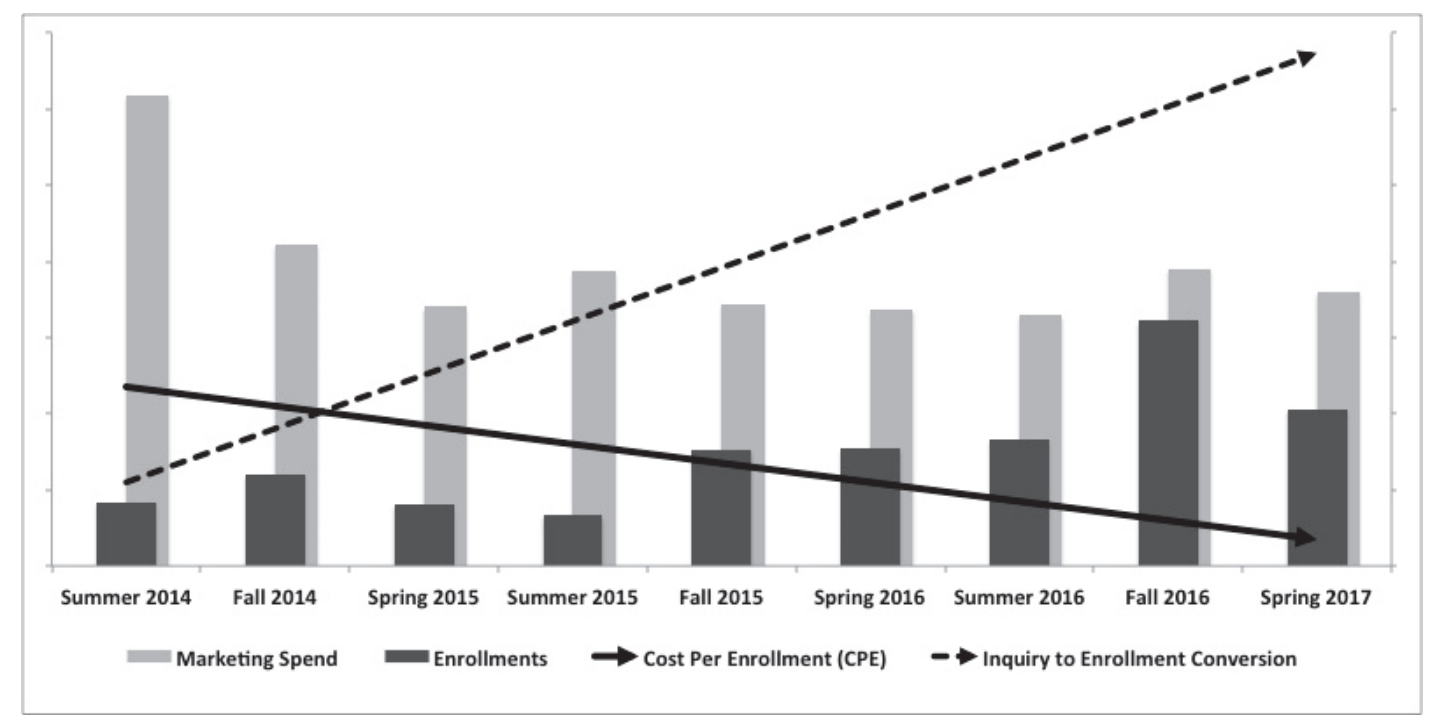

Chart 9. Enrollment versus Marketing Spend

\section{Limitations}

The limitations of the VSM case include that the process is a narrowly focused part of a student's educational life cycle, that active participation in the process was wholly contained within the MBA and Graduate admissions staff, and did not actively involve relevant university constituencies outside that cross-functional team, and that there are external forces and regulatory considerations which must be taken into account which can sometimes be difficult to navigate. 
Challenges exist as MBA recruiting is just one part of a total university so sometimes a desired change needs to be sold to another part of the university or has to wait if it is not accepted. To mitigate these possibilities the team focuses on what can be done to find a way that meets the needs of the student. The goal is never to circumvent the approved process or jeopardize accreditation or regulations. There are always steps however small that can move the process towards perfection. Each time this occurs, the knowledge of the team member expands and their mind opens to the culture of lean thinking.

\section{Conclusions}

The success of the recruitment is that both students and university are meeting their objectives. VSM built a transparent process that allows all involved to see their current state. Moving from a system of independently managed silos to one of a pull from upstream to the registration transformed how the MBA team adds value to the student recruiting process. The team culturally knows what lean management is and embraces change as positive for all involved. Some major learning includes:

1. Cross training alleviates team stress and improves student satisfaction because they do not have to wait for a response that is person dependent.

2. Pipeline analysis determines historical yield and allows for more accurate forecasting and planning.

3. Clear visibility to the MBA enrollment process and the ability to use to data to pinpoint future opportunities for improvement.

4. Incremental gains in process efficiency times but utilization of data has allowed for a clearer understanding of the future opportunity areas.

Using VSM has been very successful at UNA for our students and the COB. It takes diligent, regular focus on the students, team and data to build successful results as have been achieved. UNA's digital marketing partner commends the results and notes that through the use of VSM the enrollment results versus spend is well above what is normally experienced by their clients. A collaborative approach moved the whole process as a system and not as individual silos, which is key to the success. This work is certainly transferable to any educational institution willing to embrace VSM and make changes that create positive results for students, faculty and staff. Overall VSM and lean resulted in transforming thinking and doing. The team members would all agree that there is no going back to the old ways. And the rest of the University is beginning to ask lots of questions about what is going on to get these dramatic results.

\section{Acknowledgments}

Special thanks to Dean Greg Carnes, Zack Crowell, Amanda Waddell Jennifer Hogan, Ning Wang, and Hillary Coats to whom this work and results owe a great debt of gratitude.

\section{References}

Graduate Management Admission Council. (2015). mba.com Prospective Students Survey. GMAC.

Martin, K. (2014). Value Stream Mapping. McGraw-Hill Education.

Womack, J., \& Jones, D. (1996). Lean Thinking. Simon and Schuster. 